\title{
White Opaque Substance, a New Optical Marker on Magnifying Endoscopy: Usefulness in Diagnosing Colorectal Epithelial Neoplasms
}

\author{
Kazutomo Yamasaki', Takashi Hisabe ${ }^{1}$, Kenshi Yao ${ }^{2}$, Hiroshi Ishihara', Kentaro Imamura', Tatsuhisa Yasaka', Hiroshi Tanabe ${ }^{3}$, \\ Akinori Iwashita $^{3}$ and Toshiharu Ueki ${ }^{1}$ \\ Departments of ${ }^{1}$ Gastroenterology, ${ }^{2}$ Endoscopy, ${ }^{3}$ Pathology, Fukuoka University Chikushi Hospital, Fukuoka, Japan
}

Background/Aims: A white substance that is opaque to endoscopic light is sometimes observed in the epithelium during narrowband imaging with magnifying endoscopy of gastric or colorectal epithelial neoplasms. This prospective observational study aimed to determine whether the morphology of the white opaque substance (WOS) allows differential diagnosis between colorectal adenoma and carcinoma.

Methods: A consecutive series of patients with colorectal adenomas or early carcinomas who underwent endoscopic resection or surgical excision were studied. The morphology of the WOS was determined based on endoscopic images before the histopathological diagnosis was performed. The primary outcome was the diagnostic performance of an irregular WOS as a marker of colorectal carcinoma.

Results: The study analyzed 125 lesions. A total of 33 lesions showed an irregular WOS, and 92 lesions showed a regular WOS. Among the 33 lesions found to show an irregular WOS, 30 were carcinomas. Among the 92 lesions showing a regular WOS, 79 were adenomas. With irregular WOS as a marker of carcinoma, the diagnostic accuracy was $87 \%$, sensitivity was $91 \%$, and specificity was $86 \%$.

Conclusions: This study demonstrated the potential usefulness of the morphology of the WOS as a marker for the differential diagnosis between adenoma and carcinoma in cases of colorectal epithelial neoplasms. Clin Endosc 2021;54:570-577

Key Words: Colorectal neoplasms; Magnifying endoscopy; Narrow band imaging; White opaque substance

\section{INTRODUCTION}

Narrow-band imaging (NBI) with magnifying endoscopy (ME) has been widely applied clinically in the endoscopic diagnosis of diseases of the digestive tract in recent years. ${ }^{1-10}$ NBI utilizes an optical filter with narrow bandwidths of two different central wavelengths (415 $\mathrm{nm}$ and $540 \mathrm{~nm}$ ). NBI with

Received: July 30, 2020 Revised: October 7, 2020

Accepted: October 22, 2020

Correspondence: Takashi Hisabe

Department of Gastroenterology, Fukuoka University Chikushi Hospital, 1-1-1 Zokumyoin, Chikushino, Fukuoka 818-8502, Japan

Tel: +81-92-921-1011, Fax: +81-92-928-0856, E-mail: hisabe@cis.fukuoka-u.ac.jp ORCID: https://orcid.org/0000-0001-7308-1030

This study was presented at the 25th United European Gastroenterology Week.

cc This is an Open Access article distributed under the terms of the Creative Commons Attribution Non-Commercial License (http://creativecommons.org/ licenses/by-nc/3.0) which permits unrestricted non-commercial use, distribution, and reproduction in any medium, provided the original work is properly cited.
ME can clearly visualize both the subepithelial microvascular architecture and the microsurface structure. ${ }^{5}$ Yao et al. discovered that during NBI with ME of gastric epithelial neoplasms and chronically inflamed gastric mucosa, a white substance opaque to endoscopic light is present within the epithelium and obscures the subepithelial microvascular pattern. ${ }^{1,11,12}$ They referred to this white substance as a "white opaque substance (WOS)" in their reports. ${ }^{1,11,12}$ Furthermore, they reported that the WOS in gastric cancer shows an irregular morphology; however, that in adenoma shows a regular morphology, thus making the morphological finding of WOS a potentially useful marker for establishing the differential diagnosis between adenoma and early carcinoma (intramucosal or submucosal $[\mathrm{SM}]$ invasive carcinoma). ${ }^{11}$ Subsequent histopathological studies demonstrated that the WOS comprises minute lipid droplets accumulated within the mucosal epithelium of gastric neoplasms. ${ }^{13,14}$ Moreover, in NBI with ME of colorectal epithelial neoplasms, the subepithelial microvessels are also 
obscured by the WOS in some cases. Hisabe et al. reported the presence of WOS in colorectal epithelial neoplasm $\mathrm{s}^{15}$ and at a high prevalence in hyperplastic polyps as well. ${ }^{16}$ Subsequently, Imamura et al. reported that the WOS in the colon, as in the stomach, comprises minute lipid droplets accumulated in the neoplastic epithelium. ${ }^{17}$ However, there have been few reports on the clinical significance of colorectal WOS and no prospective studies on the morphology of colorectal WOS as a potential endoscopic marker for establishing a differential diagnosis between adenoma and early carcinoma. Thus, a prospective study was conducted to determine whether the morphology of the WOS facilitates differential diagnosis between adenoma and early carcinoma.

\section{MATERIALS AND METHODS}

\section{Patients}

All patients who underwent colonoscopy at the Fukuoka University Chikushi Hospital who were scheduled to undergo colorectal endoscopic resection or surgical excision of a colorectal epithelial neoplasm (adenoma or early carcinoma) were included, excluding those with: (1) inflammatory bowel diseases; (2) advanced cancer; (3) prior colorectal surgery; (4) familial adenomatous polyposis; (5) serious underlying disorders; (6) lesions without a histopathological diagnosis of adenoma or carcinoma; and (7) poor preparation or bleeding, among other things, that resulted in difficulty with observation.

No target sample size could be established because there have been no reports of any study on the morphology of the WOS in colorectal epithelial neoplasms conducted with the same design as in the present study. Thus, it was decided to gather cases over approximately a year between December 2015 and November 2016. Given our hospital's record of performing colorectal endoscopic treatment for about $500 \mathrm{pa}-$ tients annually, approximately 350 cases were anticipated.

This study was approved by the Medical Ethics Committee of the Fukuoka University Chikushi Hospital (R15-025) and was registered with the University Hospital Medical Information Network (UMIN 000021167). The study was conducted with ethical considerations carefully taken into account, and with each patient personally receiving extensive information of the study on a patient information sheet and providing informed consent by signing and dating an informed consent form prior to enrollment.

A policy for the complete removal of neoplastic lesions that were detected was adopted. In cases in which a lesion was observed, the surface of the neoplasm was rinsed with water, and the entire lesion was examined using NBI with ME, with the endoscopic images captured and recorded in a filing system. The lesion was endoscopically resected if endoscopic treatment was deemed possible or surgically excised if surgery was deemed necessary.

The presence or absence of the WOS was determined based solely on the magnifying NBI endoscopic images saved in the patient filing system before the pathological diagnosis was confirmed. Two independent endoscopists, one experienced (number of colonoscopies performed $\geq 5,000[\mathrm{TH}]$ ) and the other inexperienced (number of colonoscopies performed $<5,000[\mathrm{HI}]$ ), reviewed all the endoscopic images.

Neoplasms were classified macroscopically based on the Paris classification. ${ }^{18}$ The location of each neoplasm was classified as right colon (cecum to transverse colon), left colon (descending colon to sigmoid colon), or rectum. The histopathological findings of the resected lesions were considered the gold standard in this study.

\section{Endoscopic specification and procedures}

All patients were administered 2-3 L of polyethylene glycol-electrolyte solution in the morning on the day of the examination as preparation. Scopolamine butylbromide or glucagon was administered in the absence of contraindications, and midazolam was used for conscious sedation only when a patient complained of discomfort or pain.

The endoscopies were performed using an electronic endoscopy system (EVIS LUCERA ELITE; Olympus Co., Ltd., Tokyo, Japan) with magnifying colonoscopy (PCF-Q240AZI, PCF-Q260AZI, CF-H260AZI, CF-HQ290AZI; Olympus). The NBI with ME observation condition was set to structure enhancement A8. The endoscopic examinations were conducted with a black soft rubber hood mounted at the tip of the endoscope for magnified observation. The lesion was visualized using incremental movements of the tip of the endoscope to bring the image into focus, with a distally attached soft black hood to stabilize the tip of the endoscope without causing mucosal injury.

\section{Definition of white opaque substance-positive and its morphology}

WOS-positive was defined as the presence of a WOS in any part of a lesion on NBI with ME. Images of WOS-negative and WOS-positive lesions are shown in Fig. 1.

The WOS-positive lesions in which the WOS was present in no less than half of the area under maximal magnification were evaluated to determine whether the WOS showed a regular or irregular morphology (Fig. 2). It is challenging to assess the morphology of WOS in cases where the WOS is present 

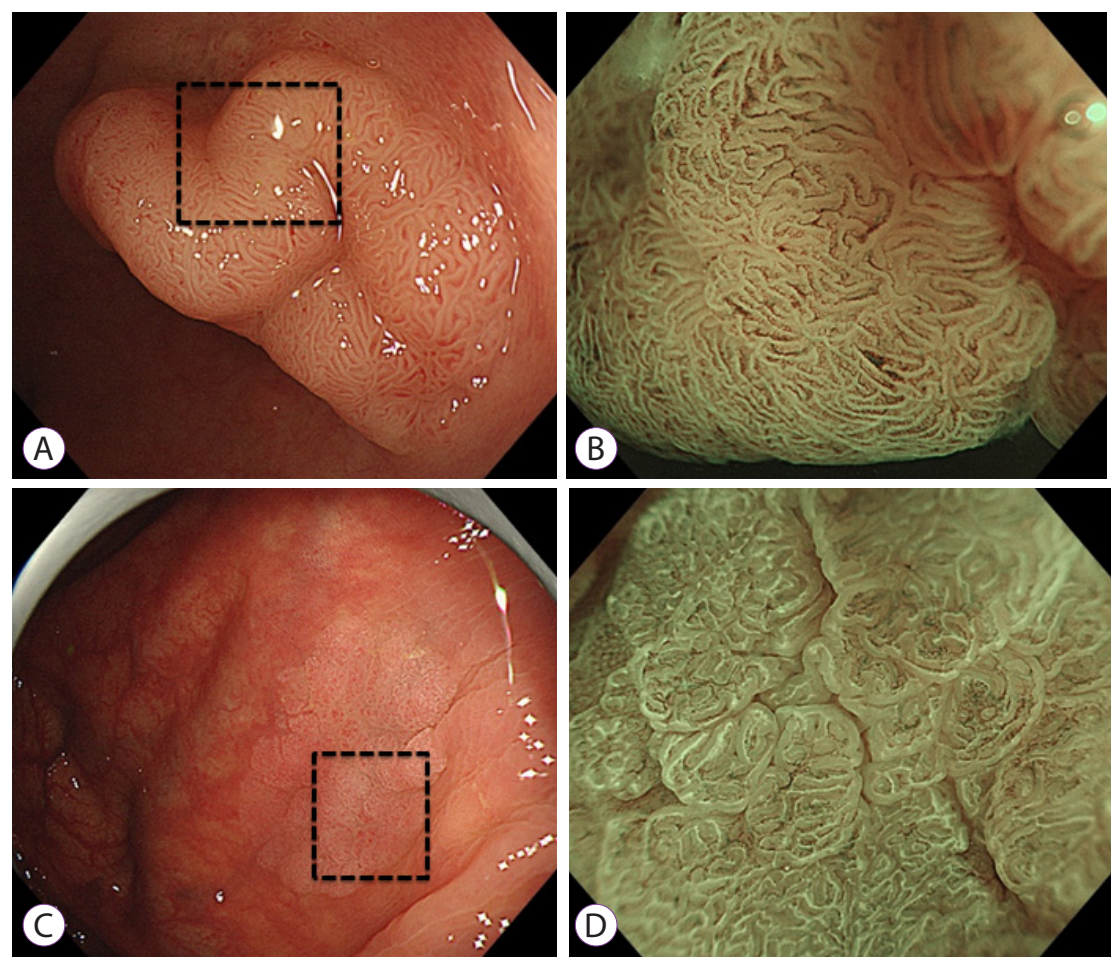

Fig. 1. Endoscopic findings of white opaque substance (WOS)-positive and WOS-negative colorectal epithelial neoplasms. (A) Image of a WOS-negative colorectal epithelial neoplasm observed with white light. (B) Magnifying narrow-band imaging (NBI) endoscopic view of the square area. The WOS is absent, with the microvessels clearly visible. (C) Image of a WOS-positive colorectal epithelial neoplasm observed with white light. (D) Magnifying NBI endoscopic view of the square area. The WOS is present, with the microvessels practically obscured.
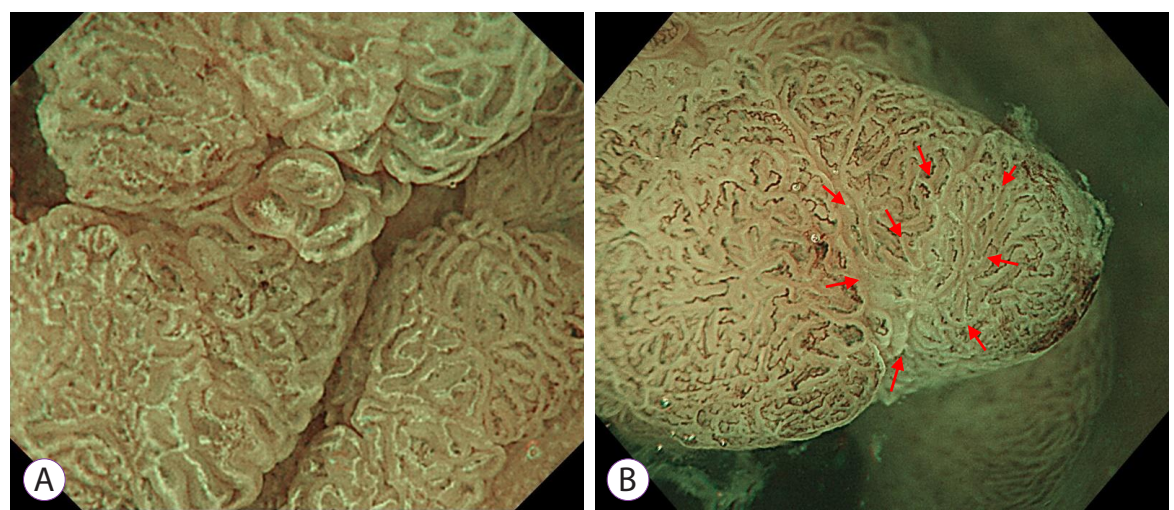

Fig. 2. Determination of whether a lesion is white opaque substance (WOS)-positive. (A) The morphology of the WOS can be visualized. The WOS is present in no less than half of the area when visualized under maximal magnification. (B) The morphology of the WOS cannot be evaluated. Although the WOS is seen in the areas identified by red arrows when visualized under maximal magnification, it is present in less than half of the overall area.

over less than half of the area under maximal magnification. In such cases, we can assess the microvascular pattern and the microsurface pattern as markers for magnifying endoscopic diagnosis of neoplasms.

Morphological findings of the WOS were classified as reg- ular or irregular according to the report by Yao et al. ${ }^{11}$ and as defined below.

A regular WOS was defined as a well-organized and symmetrical distribution of the dense WOS in a regular reticular, maze-like, or speckled pattern. 


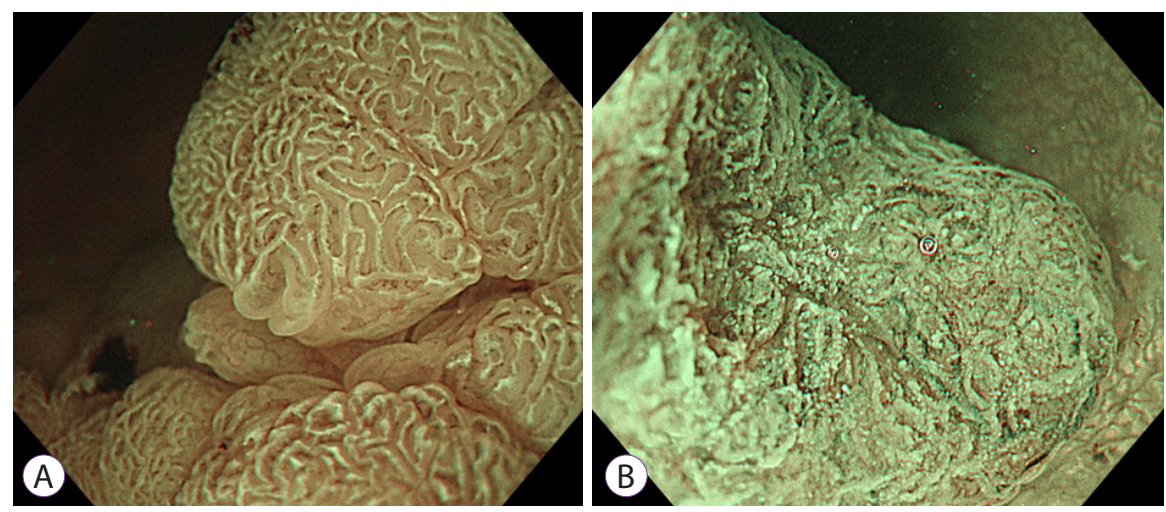

Fig. 3. Morphological findings of the white opaque substance (WOS). (A) Regular WOS: morphology of the WOS shows a well-organized and symmetrical distribution of a regular reticular pattern. (B) Irregular WOS: morphology of the WOS shows a disorganized and asymmetrical distribution of an irregular speckled pattern.

An irregular WOS was defined as a disorganized and asymmetrical distribution of the fine WOS in an irregular reticular, speckled pattern.

Images of irregular WOS and regular WOS lesions are shown in Fig. 3.

\section{Histopathological investigations}

Resected specimens were mounted on a plate and sliced at 2 -mm intervals after overnight fixation in a $20 \%$ buffered formalin solution. These specimens were embedded in paraffin and cut into $5-\mu \mathrm{m}$-thick tissue sections. The sections were subsequently stained with hematoxylin and eosin. Histopathological findings of resected specimens classified as Category 3 (low-grade adenoma) and those classified as Category 4 (highgrade adenoma, a non-invasive carcinoma, or intramucosal carcinoma) or Category 5 (carcinoma invading the submucosa or beyond) according to the revised Vienna classification ${ }^{19}$ were reclassified as adenoma and carcinoma, respectively.

Neoplasms with shallow SM invasion were defined as SM-s cancer (the depth of SM invasion was limited to less than 1,000 $\mu \mathrm{m}$ ), and those with deep SM invasion were defined as SM-d cancer (the depth of SM invasion was over 1,000 $\mu \mathrm{m}$ ).

\section{Outcome measures}

The primary outcome was the diagnostic performance (accuracy, sensitivity, specificity, positive predictive value [PPV], and negative predictive value [NPV]) of the irregular WOS as a marker of colorectal carcinoma in colorectal epithelial neoplasms (adenomas and early carcinomas).

The secondary outcomes were as follows: (1) differences in the prevalence of an irregular WOS by the depth of invasion in early colorectal carcinoma; (2) comparison of the presence/ absence of lymph node metastases between regular and irreg- ular WOS-positive lesions in early colorectal carcinoma; and (3) the intraobserver agreement (TH) and the interobserver agreement (TH and $\mathrm{HI}$ ), with the second evaluation, performed 2 months later on the same endoscopic images that had been randomized (TH).

\section{Statistical analysis}

Comparisons of the prevalence between the two groups were made using Fisher's exact test or Pearson's chi-square test. $P$-values $<0.05$ were considered significant. SPSS version 21 J for Windows (IBM SPSS Statistics, Chicago, IL, USA) was used for all statistical analyses.

\section{RESULTS}

\section{Recruitment and participant flow}

A total of 511 lesions were identified in 296 patients by colorectal endoscopy over a 1-year period from December 2015 to November 2016 for inclusion in the study. Of these lesions, 30 could not be evaluated due to mucous or other reasons, and 268 WOS-negative lesions (252 adenomas and 16 early carcinomas) were excluded. The WOS was present in 213 lesions, including 145 adenomas, 52 early carcinomas, and 16 hyperplastic polyps or sessile serrated adenomas/polyps (SSA/ Ps). The prevalence of WOS-positivity was $100 \%(16 / 16)$ in hyperplastic polyps, $36.5 \%$ (145/397) in adenomas, and 76.5\% $(52 / 68)$ in early carcinomas. Of the 213 lesions, 16 were diagnosed as hyperplastic polyps or SSA/Ps, and 72 lesions with the WOS present over less than half of the area under maximal magnification were excluded. The remaining 125 lesions in 96 patients were analyzed (Fig. 4). 


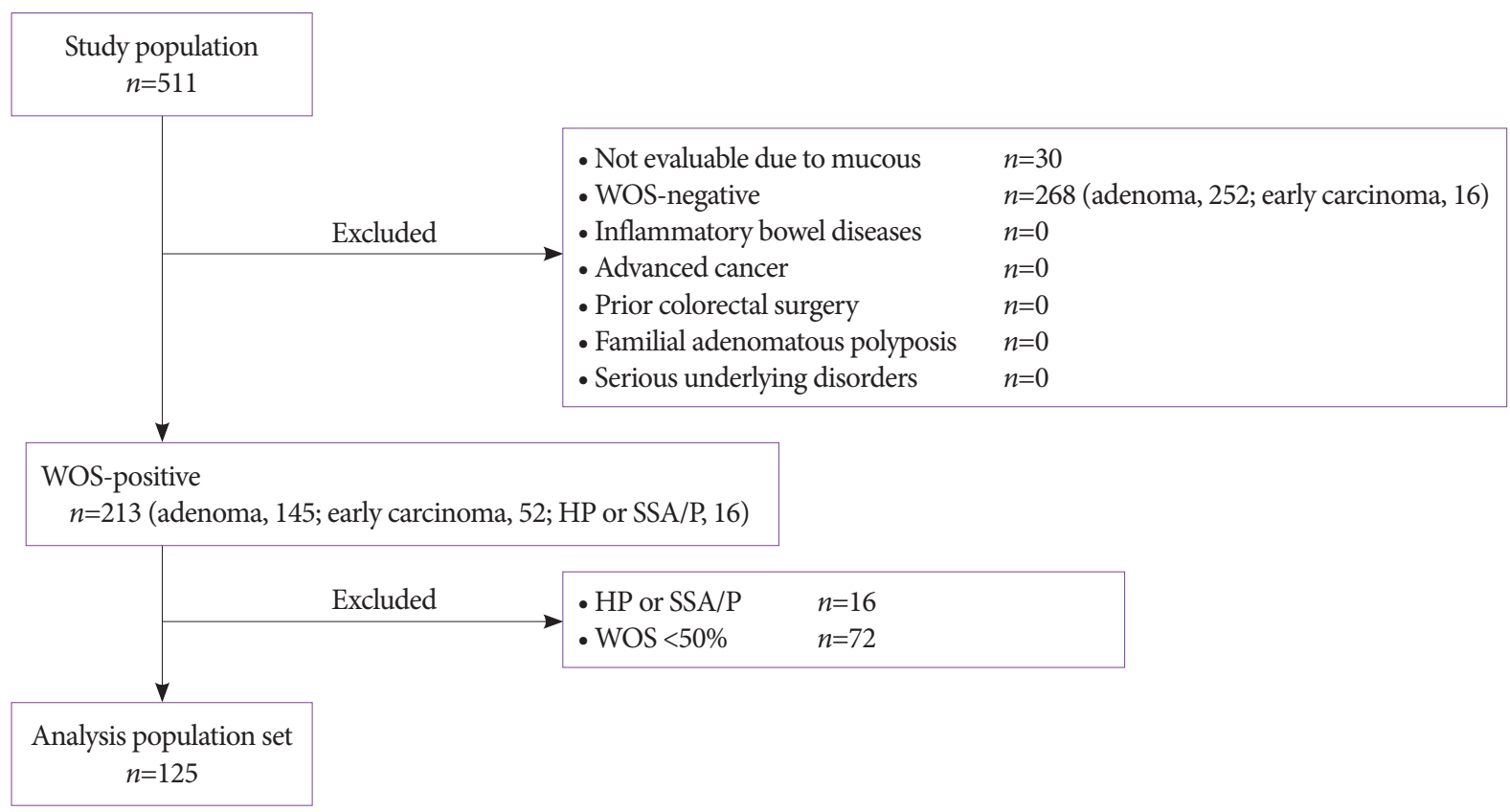

Fig. 4. Participant flow. HP, hyperplastic polyp; SSA/P, sessile serrated adenoma/polyp; WOS, white opaque substance.

\section{Clinicopathological features}

The 96 patients included 52 men and 44 women, with a mean age of 64.4 years at diagnosis. Lesion characteristics are presented in Table 1. The mean tumor size was $14.0 \mathrm{~mm}$, with 52 of the lesions located in the right colon, 49 in the left colon, and 24 in the rectum. The macroscopic classification of the neoplasms were 0 -IIa, 0 -IIc, or 0-IIa +IIc in 34 lesions and 0-Is, 0-Isp, or 0-Ip in 91 lesions. Furthermore, the 125 lesions included 82 adenomas and 43 early carcinomas.

Table 1. Clinicopathological Findings of the Analyzed Lesions ( $n=125)$

\begin{tabular}{ll}
\hline Tumor size (mean size \pm SD, mm) & $14.0 \pm 10.0$ \\
Lesion location & \\
Right colon & $52(41.6 \%)$ \\
Left colon & $49(39.2 \%)$ \\
Rectum & $24(19.2 \%)$ \\
Macroscopic classification & \\
0-IIa, IIc, IIa+IIc & $34(27.2 \%)$ \\
0-Is, Isp, Ip & $91(72.8 \%)$ \\
Histologic type & \\
Adenoma & $82(65.6 \%)$ \\
Intramucosal carcinoma & $29(23.2 \%)$ \\
Submucosal invasive carcinoma & $14(11.2 \%)$ \\
\hline
\end{tabular}

$\mathrm{SD}$, standard deviation.

\section{Outcomes}

\section{Diagnostic performance}

A total of 3/82 lesions (3.7\%) showed an irregular WOS, while $79 / 82$ lesions $(96.3 \%)$ showed a regular WOS. Of the 43 early carcinomas, 30 lesions (69.8\%) showed an irregular WOS, and 13 lesions (30.2\%) showed a regular WOS. The prevalence of an irregular WOS was significantly higher in early carcinomas than in adenomas $(p<0.001$, Fisher's exact test; Table 2). With an irregular WOS as a marker of early carcinoma in the differential diagnosis between early carcinoma and adenoma, the sensitivity was $91 \%$, specificity was $86 \%$, PPV was $70 \%$, NPV was 96\%, and accuracy was $87 \%$ (Table 3).

Table 2. Prevalences of a Regular White Opaque Substance and an Irregular White Opaque Substance in Colorectal Adenomas and Early Carcinomas

\begin{tabular}{lccc}
\hline & $\begin{array}{c}\text { Irregular WOS } \\
\boldsymbol{n = 3 3}\end{array}$ & $\begin{array}{c}\text { Regular WOS } \\
\boldsymbol{n = 9 2}\end{array}$ & $\boldsymbol{p}$-value \\
\hline Early carcinoma & & & \\
$n=43$ & $30(69.8 \%)$ & $13(30.2 \%)$ & \\
$\begin{array}{l}\text { Adenoma } \\
n=82\end{array}$ & $3(3.7 \%)$ & $79(96.3 \%)$ & $<0.001$ \\
\hline
\end{tabular}

Pearson Chi-Square test.

WOS, white opaque substance. 
Table 3. Diagnostic Performance with the Irregular White Opaque Substance as a Marker of Carcinoma

\begin{tabular}{ccccc}
\hline $\begin{array}{c}\text { Sensitivity } \\
(\mathbf{9 5 \%} \mathbf{C I})\end{array}$ & $\begin{array}{c}\text { Specificity } \\
(\mathbf{9 5 \%} \mathbf{C I})\end{array}$ & $\begin{array}{c}\text { PPV } \\
(\mathbf{9 5 \%} \mathbf{C I})\end{array}$ & $\begin{array}{c}\text { NPV } \\
\mathbf{( 9 5 \% ~ C I )}\end{array}$ & $\begin{array}{c}\text { Accuracy } \\
(\mathbf{9 5 \%} \mathbf{C I})\end{array}$ \\
\hline 0.91 & 0.86 & 0.70 & 0.96 & 0.87 \\
$(0.81-1.00)$ & $(0.78-0.92)$ & $(0.56-0.83)$ & $(0.92-1.00)$ & $(0.81-0.93)$ \\
\hline
\end{tabular}

CI, confidence interval; NPV, negative predictive value; PPV, positive predictive value.

Table 4. Clinicopathological Findings of Early Carcinomas by White Opaque Substance Morphology

\begin{tabular}{|c|c|c|c|}
\hline & $\begin{array}{c}\text { Irregular WOS } \\
\qquad n=30\end{array}$ & $\begin{array}{c}\text { Regular WOS } \\
n=13\end{array}$ & $p$-value \\
\hline Neoplasm size (mean size $\pm \mathrm{SD}, \mathrm{mm}$ ) & $19.8 \pm 10.6$ & $20.5 \pm 14.4$ & 0.541 \\
\hline \multicolumn{4}{|l|}{ Lesion location } \\
\hline Right colon & 9 & 5 & 0.418 \\
\hline Left colon & 12 & 4 & 0.413 \\
\hline Rectum & 9 & 4 & 0.615 \\
\hline \multicolumn{4}{|l|}{ Macroscopic classification } \\
\hline 0-IIa, 0-IIc, 0-IIa+IIc & 12 & 4 & 0.414 \\
\hline 0-Is, 0-Isp, 0-Ip & 18 & 9 & \\
\hline \multicolumn{4}{|l|}{ Depth of invasion } \\
\hline Intaramucosal carcinoma, SM-s carcinoma & 21 & 10 & 0.727 \\
\hline SM-d carcinoma & 9 & 3 & \\
\hline
\end{tabular}

Fisher's exact test.

SD, standard deviation; SM-d, the depth of submucosal invasion is over 1,000 $\mu \mathrm{m}$; SM-s, the depth of submucosal invasion is limited to less than $1,000 \mu \mathrm{m}$; WOS, white opaque substance.

\section{Clinicopathological findings by white opaque substance morphology}

Mean neoplasm sizes, lesion locations, macroscopic classifications, and depths of invasion of the early carcinomas are presented by the WOS morphology in Table 4. In early carcinomas, lesions with a regular WOS and lesions with an irregular WOS showed no significant difference in terms of mean neoplasm size, lesion location, and macroscopic classification. By the depth of invasion of an early carcinoma, 21/31 intramucosal carcinomas or SM-s carcinomas (67.8\%) showed an irregular WOS, while 9/12 SM-d carcinomas (75\%) showed an irregular WOS, indicating no significant difference in the prevalence of an irregular WOS between intramucosal carcinomas or SM-s carcinomas and SM-d carcinomas $(p=0.727)$ (Table 4). A total of 8/12 SM-d carcinomas underwent surgical treatment; however, only one with a regular WOS was confirmed to have lymph node metastasis.

\section{Intraobserver and interobserver agreement}

For WOS morphology, the interobserver agreement $(\mathrm{TH}$ and HI) was categorized as moderate $(\kappa=0.587)$, whereas the intraobserver agreement $(\mathrm{TH})$ was categorized as good to fair $(\kappa=0.653)$.

\section{DISCUSSION}

The present study demonstrated the usefulness of the morphology of the WOS, a new optical marker for NBI with ME, for differentiating between adenoma and early carcinoma in colorectal epithelial neoplasms.

Hisabe et al. ${ }^{15}$ reported the presence of the WOS in approximately $40 \%$ of colorectal epithelial neoplasms, and that the prevalence of WOS-positivity was significantly higher in early carcinomas than in adenomas (66\% vs. $31.8 \%$ ), and it was significantly higher in SM carcinomas than in intramucosal 
carcinomas ( $75.9 \%$ vs. $59.0 \%)$. The present study provided similar results; the prevalence of WOS-positivity was $36.5 \%$ in adenomas and $76.5 \%$ in early carcinomas. Nevertheless, it is still challenging to differentiate between carcinomas and adenomas based on the presence or absence of the WOS alone. Yao et al. ${ }^{11}$ proposed an algorithm for cases of gastric epithelial neoplasms with the WOS obscuring the microvessels; magnifying endoscopic diagnosis should be made alternatively with WOS morphology as a marker of the microsurface structure. In cases of gastric epithelial neoplasms with the extensive presence of the WOS, using an irregular WOS as a marker of carcinoma has allowed differentiation between carcinoma and adenoma with a sensitivity of $97.7 \%$ and a specificity of $96.0 \%$, making it possible to determine whether a lesion is carcinomatous. Similarly, the analysis of the morphology of the WOS seems to be useful for an accurate diagnosis, as demonstrated in this study.

A retrospective study of the morphology of the WOS in colorectal epithelial neoplasms reported that among 71 lesions with a regular WOS, adenomas accounted for $54.9 \%$, and high-grade dysplasias (HGDs) and SM-s carcinomas accounted for $43.7 \%$, indicating that the regular WOS is a marker for adenoma or HGDs/SM-s carcinomas. ${ }^{20}$ The present study, on the other hand, showed different results; specifically, a regular WOS is characteristic of adenomas, while an irregular WOS is a characteristic finding associated with HGDs and SM-s carcinomas. In fact, the prevalence of an irregular WOS was significantly higher in early carcinomas than in adenomas ( $69.8 \%$ vs. $3.7 \%$, respectively, $p<0.001)$. In the differential diagnosis between early carcinoma and adenoma, the use of the irregular WOS as a marker of early carcinoma provided high sensitivity (91\%), specificity (86\%), and accuracy (87\%). As seen with gastric epithelial neoplasms, the WOS is also present extensively in colorectal epithelial neoplasms. In cases where the WOS obscures the vessel pattern, it is important to overlook the vessel pattern and use the morphology of the WOS as an alternative surface-pattern marker to establish a magnifying endoscopic diagnosis. However, in our study, three cases with irregular WOS had established the diagnosis of adenoma, and 13 cases with regular WOS had established the diagnosis of early carcinoma. Even in early carcinoma, it is challenging to evaluate the morphology of WOS as irregular if the lesion has a weak histological and structural atypia on the surface. On the other hand, adenoma with strong structural atypia on the surface may be evaluated as malformed.

A retrospective study by Kawasaki et al. ${ }^{20}$ on WOS morphology stratified by the depth of invasion of the carcinoma showed that irregular WOS was present in $82.4 \%$ of carcinomas that had invaded deeply into the SM layer and in $14.7 \%$ of HGDs and SM-s carcinomas, indicating that the irregular
WOS is a marker of deep SM invasive carcinoma. Furthermore, the risk of lymph node metastasis has been higher in deep SM invasive carcinomas with irregular WOS than in those without WOS. In the present study, however, $67.8 \%$ of intramucosal or SM-s carcinomas and $75 \%$ of SM-d carcinomas showed an irregular WOS, indicating no significant difference in the prevalence of irregular WOS. Thus, it is challenging to diagnose the depth of invasion of a carcinoma based on the WOS morphology, and cases with a confirmed irregular WOS must be subsequently evaluated by pit pattern ${ }^{21}$ diagnosis using chromoendoscopy. Moreover, none of the SM-d carcinomas positive for an irregular WOS had lymph node metastasis, showing no relationship between lymph node metastasis and irregular WOS. We speculate that the retrospective review of the recorded endoscopic images has limitations for assessing the prevalence or the morphology of WOS because the WOS is not uniformly present in a diffuse manner in one neoplasm but rather in a patchy manner on the surface of the neoplasm. When we take into consideration such limitations, the data obtained from this prospective study seems to be more reliable because prospective endoscopic observation can be more sensitive towards a WOS that is present in a patchy manner.

There are some limitations to this study. First, it was a single-site study in which only two endoscopists performed the evaluations. The $\kappa$-value in the study was moderate for interobserver agreement and good to fair for intraobserver agreement, showing good reproducibility in determining the WOS morphology. Nonetheless, a multicenter study involving many endoscopists is needed. Moreover, colorectal epithelial neoplasms include carcinomas in adenoma; however, this study did not evaluate whether the part evaluated for the WOS under NBI with ME was consistent with the part showing a high histopathological grade.

It is important to note, however, that the present study demonstrated in a prospective cohort of patients that the morphology of the WOS in colorectal epithelial neoplasms has the potential to serve as a highly reproducible marker in endoscopic procedures. Presently, the NBI classification, ${ }^{3,22-26}$ including the Japan NBI Expert Team (JNET) classification, does not provide any criteria about the morphology of the WOS. Given the considerable number of cases with the WOS obscuring the vessel pattern, the morphological finding of the WOS has the potential to become a surface pattern marker.

In conclusion, this study demonstrated the potential usefulness of the morphology of the WOS on NBI with ME as a marker in the differential diagnosis between carcinoma and adenoma in cases of colorectal epithelial neoplasm with the WOS obscuring the microvessels. 
Conflicts of Interest

The authors have no potential conflicts of interest.

Funding

None.

$\begin{array}{ll}\text { ORCID } & \\ \text { Kazutomo Yamasaki: } & \text { https://orcid.org/0000-0003-2501-0726 } \\ \text { Takashi Hisabe: } & \text { https://orcid.org/0000-0001-7308-1030 } \\ \text { Kenshi Yao: } & \text { https://orcid.org/0000-0003-0863-3649 } \\ \text { Hiroshi Ishihara: } & \text { https://orcid.org/0000-0003-3236-5322 } \\ \text { Kentaro Imamura: } & \text { https://orcid.org/0000-0003-2307-841X } \\ \text { Tatsuhisa Yasaka: } & \text { https://orcid.org/0000-0002-9115-988X } \\ \text { Hiroshi Tanabe: } & \text { https://orcid.org/0000-0003-3776-5819 } \\ \text { Akinori Iwashita: } & \text { https://orcid.org/0000-0002-8095-5987 } \\ \text { Toshiharu Ueki: } & \text { https://orcid.org/0000-0002-4142-5895 }\end{array}$

\section{REFERENCES}

1. Yao K, Anagnostopoulos GK, Ragunath K. Magnifying endoscopy for diagnosing and delineating early gastric cancer. Endoscopy 2009;41:462467.

2. Sano Y, Ikematsu H, Fu KI, et al. Meshed capillary vessels by use of narrow-band imaging for differential diagnosis of small colorectal polyps. Gastrointest Endosc 2009;69:278-283.

3. Kanao H, Tanaka S, Oka S, Hirata M, Yoshida S, Chayama K. Narrow-band imaging magnification predicts the histology and invasion depth of colorectal tumors. Gastrointest Endosc 2009;69(3 Pt 2):631-636.

4. Ezoe Y, Muto M, Uedo N, et al. Magnifying narrowband imaging is more accurate than conventional white-light imaging in diagnosis of gastric mucosal cancer. Gastroenterology 2011;141:2017-2025.e3.

5. Machida H, Sano Y, Hamamoto Y, et al. Narrow-band imaging in the diagnosis of colorectal mucosal lesions: a pilot study. Endoscopy 2004:36:1094-1098.

6. East JE, Suzuki N, Bassett P, et al. Narrow band imaging with magnification for the characterization of small and diminutive colonic polyps: pit pattern and vascular pattern intensity. Endoscopy 2008;40:811-817.

7. Sikka S, Ringold DA, Jonnalagadda S, Banerjee B. Comparison of white light and narrow band high definition images in predicting colon polyp histology, using standard colonoscopes without optical magnification. Endoscopy 2008;40:818-822.

8. Hewett DG, Kaltenbach T, Sano Y, et al. Validation of a simple classification system for endoscopic diagnosis of small colorectal polyps using narrow-band imaging. Gastroenterology 2012;143:599-607.e1.

9. Wani S, Rastogi A. Narrow-band imaging in the prediction of submucosal invasive colon cancer: how "NICE" is it? Gastrointest Endosc 2013;78:633-636.

10. Singh R, Nordeen N, Mei SL, Kaffes A, Tam W, Saito Y. West meets East: preliminary results of narrow band imaging with optical magnification in the diagnosis of colorectal lesions: a multicenter Australian study using the modified Sano's classification. Dig Endosc 2011;23 Suppl 1:126130.

11. Yao K, Iwashita A, Tanabe H, et al. White opaque substance within su- perficial elevated gastric neoplasia as visualized by magnification endoscopy with narrow-band imaging: a new optical sign for differentiating between adenoma and carcinoma. Gastrointest Endosc 2008;68:574580 .

12. Kanemitsu T, Yao K, Nagahama T, et al. Extending magnifying NBI diagnosis of intestinal metaplasia in the stomach: the white opaque substance marker. Endoscopy 2017;49:529-535.

13. Yao K, Iwashita A, Nambu M, et al. Nature of white opaque substance in gastric epithelial neoplasia as visualized by magnifying endoscopy with narrow-band imaging. Dig Endosc 2012;24:419-425.

14. Ueo T, Yonemasu H, Yada N, et al. White opaque substance represents an intracytoplasmic accumulation of lipid droplets: immunohistochemical and immunoelectron microscopic investigation of 26 cases. Dig Endosc 2013;25:147-155.

15. Hisabe T, Yao K, Imamura K, et al. White opaque substance visualized using magnifying endoscopy with narrow-band imaging in colorectal epithelial neoplasms. Dig Dis Sci 2014;59:2544-2549.

16. Hisabe T, Yao K, Imamura K, et al. Novel endoscopic findings as visualized by magnifying endoscopy with narrow-band imaging: white opaque substance is present in colorectal hyperplastic polyps. Digestion 2016;93:127-131.

17. Imamura $\mathrm{K}$, Yao K, Hisabe $\mathrm{T}$, et al. The nature of the white opaque substance within colorectal neoplastic epithelium as visualized by magnifying endoscopy with narrow-band imaging. Endosc Int Open 2016;4:E1151-E1157.

18. The Paris endoscopic classification of superficial neoplastic lesions: esophagus, stomach, and colon: November 30 to December 1, 2002. Gastrointest Endosc 2003;58(6 Suppl):S3-S43.

19. Schlemper RJ, Kato Y, Stolte M. Diagnostic criteria for gastrointestinal carcinomas in Japan and Western countries: proposal for a new classification system of gastrointestinal epithelial neoplasia. J Gastroenterol Hepatol 2000;15 Suppl:G49-G57.

20. Kawasaki K, Kurahara K, Yanai S, et al. Significance of a white opaque substance under magnifying narrow-band imaging colonoscopy for the diagnosis of colorectal epithelial neoplasms. Gastrointest Endosc 2015;82:1097-1104.

21. Kudo S, Tamura S, Nakajima T, Yamano H, Kusaka H, Watanabe H. Diagnosis of colorectal tumorous lesions by magnifying endoscopy. Gastrointest Endosc 1996;44:8-14.

22. Tanaka S, Sano Y. Aim to unify the narrow band imaging (NBI) magnifying classification for colorectal tumors: current status in Japan from a summary of the consensus symposium in the 79th annual meeting of the Japan Gastroenterological Endoscopy Society. Dig Endosc 2011;23 Suppl 1:131-139.

23. Sano Y, Tanaka S, Kudo SE, et al. Narrow-band imaging (NBI) magnifying endoscopic classification of colorectal tumors proposed by the Japan NBI Expert Team. Dig Endosc 2016;28:526-533.

24. Ikematsu H, Matsuda T, Emura F, et al. Efficacy of capillary pattern type IIIA/IIIB by magnifying narrow band imaging for estimating depth of invasion of early colorectal neoplasms. BMC Gastroenterol 2010;10:33.

25. Wada Y, Kudo SE, Kashida H, et al. Diagnosis of colorectal lesions with the magnifying narrow-band imaging system. Gastrointest Endosc 2009;70:522-531.

26. Hisabe T, Yao K, Beppu T, et al. Validity of the usefulness of microvascular architecture and microsurface structure using magnifying endoscopy with narrow-band imaging in the colorectal neoplasm. Ann Gastroenterol 2013;26:45-51. 